\title{
THE NUMBER SYSTEM AFTER DEDEKIND
}

BY W. F. OSGOOD

1. Introduction. The rational numbers (positive, negative, and zero) having been defined and the four species for them established, the creation of the irrational numbers is possible in a variety of ways, notably by regular sequences and by Dedekind's cut.

The first method is, perhaps, easiest for the beginner. The fundamental principle of limits once being accepted in the form of the definition of a regular sequence, the detailed developments proceed smoothly, and the system of real numbers is evolved satisfactorily.*

Complete as this method is, there are mathematicians who feel that the definition of irrational numbers by means of the cut is essentially simpler, more elementary, for it creates these numbers before the concept of a limiting process is introduced and thus provides the elements for point sets before any infinite process has been defined.

Addition and subtraction are defined in the simplest manner possible on the basis of the cut. If $A=\left(a_{1}, a_{2}\right)$ and $B=\left(b_{1}, b_{2}\right)$ are any two numbers, then $C=A+B$ is the number

$$
\left(c_{1}, c_{2}\right)=\left(a_{1}+b_{1}, a_{2}+b_{2}\right) .
$$

But multiplication cannot be defined in the same way. One would like to define $C=A B$ as the number $\left(c_{1}, c_{2}\right)=\left(a_{1} b_{1}, a_{2} b_{2}\right)$; but this is impossible since the fractions $a_{1} b_{1}, a_{2} b_{2}$ do not yield a cut. True, one can introduce the positive irrationals after the positive fractions, and before the negative numbers and zero, have been defined. The above definition of multiplication as the product of two cuts is now possible. The definition of negative numbers goes through exactly as before, and the system of real numbers is complete. We have lost, however, the earlier definition of a real number as a cut, and this is too high a price to pay for the simplified definition of multiplication.

* Pierpont has given an excellent exposition of this method in his Functions of Real Variatles, vol. 1, pp. 31-61. 
2. The Way Out. There is, however, a way out, which is provided by the conception of the limit set forth in Mr. Hsü's paper.* It has already been remarked that as soon as the system of real numbers has been created through the definition of the cut, and $<,=,>$ have been defined, the basis for a theory of point sets has been provided, even before addition, to say nothing of multiplication, has been defined. In particular, it is possible to define the limit of a point set.

By the neighborhood of a point (=number), $A=\left(a_{1}, a_{2}\right)$, shall be meant the numbers $x$ such that

$$
\gamma_{1}<x<\gamma_{2},
$$

where $\gamma_{1}$ is a number $<A$ and $\gamma_{2}$ is a number $>A$.

By the $\epsilon$-neighborhood of $A$ shall be meant the neighborhood for which

$$
\gamma_{1}=\left(a_{1}-\epsilon, a_{2}-\epsilon\right), \quad \gamma_{2}=\left(a_{1}+\epsilon, a_{2}+\epsilon\right),
$$

where $\epsilon$ is any positive fraction.

3. Definition of Convergence. Let $M_{1}, M_{2}, \cdots$ be a succession, or sequence, of point sets. Then this sequence is said to converge if to an arbitrary fraction $\epsilon>0$ there corresponds an integer $m$ and a point $\zeta_{m}$ of the set $M_{m}$ such that the $\epsilon$-neighborhood of $\zeta_{m}$ contains all the sets $M_{n}$ for which $m \leqq n$.

Theorem 1. Let $M_{1}, M_{2}, \cdots$ be a convergent sequence. Then there exists one and only one point

$$
u=\left(u_{1}, u_{2}\right)
$$

such that an arbitrary $\epsilon$-neighborhood of $U$ contains each $M_{n}$, $m \leqq n$; and conversely.

4. Definition of a Limit. Let $M_{1}, M_{2}, \cdots$ be a convergent sequence. The number $U$ related to it by Theorem 1 is defined as the limit of the sequence:

$$
U=\lim _{n=\infty} M_{n} .
$$

Theorem 2. Let $M_{1}, M_{2}, \cdots$ be a convergent sequence. Let $N_{n}$ be composed of some or all the points of $M_{n}$. Then the sequence $N_{1}, N_{2}, \cdots$ is convergent, and its limit is equal to the limit of the sequence $M_{1}, M_{2}, \cdots$.

* This Bulletin, vol. 41 (1935), p. 502. 
5. Definition of Multiplication. Let $A=\left(a_{1}, a_{2}\right)$ and $B=\left(b_{1}, b_{2}\right)$ be any two numbers. Let

$$
\begin{aligned}
\epsilon_{1} \geqq \epsilon_{2} \geqq \cdots, & & \lim _{n=\infty} \epsilon_{n}=0 ; \\
\eta_{1} \geqq \eta_{2} \geqq \cdots, & & \lim _{n=\infty} \eta_{n}=0,
\end{aligned}
$$

be two sequences of positive fractions. Let $M_{n}$ be the point set composed of the fractions $a_{p} b_{q}$, $M_{n}$ :

$$
\left\{a_{p} b_{q}\right\}
$$

where $a_{p},(p=1,2)$, is any fraction of the $\epsilon_{n}$-neighborhood of $A$, and $b_{q},(q=1,2)$, is any fraction of the $\eta_{n}$-neighborhood of $B$. Then the sequence $M_{1}, M_{2}, \cdots$ converges, and its limit is defined as the product $A B$ :

$$
A B=\lim _{n=\infty}\left\{a_{p} b_{q}\right\} .
$$

That multiplication obeys the commutative law, $A B=B A$, is at once obvious. The associative law is easily established by means of the sequence $M_{1}^{\prime}, M_{2}^{\prime}, \cdots$, where $C=\left(c_{1}, c_{2}\right)$ is any third number and $M_{n}^{\prime}$ is composed of the fractions $a_{p} b_{q} c_{r}$, $M_{n}^{\prime}$ :

$$
\left\{a_{p} b_{q} c_{r}\right\}
$$

$c_{r},(r=1,2)$, being any fraction lying in the $\lambda_{n}$-neighborhood of $C$. Here,

$$
\lambda_{1} \geqq \lambda_{2} \geqq \cdots, \quad \lim _{n=\infty} \lambda_{n}=0,
$$

is a sequence of positive fractions. The sequence $M_{1}^{\prime}, M_{2}^{\prime}, \cdots$ converges; and now it is shown without difficulty that its limit has the value $(A B) C$. But the limit is obviously invariant of the order of the factors $a_{p}, b_{q}, c_{r}$. Hence it also has the value

$$
(B C) A=A(B C) \text {. }
$$

Division is now shown to be possible and unique, except when the divisor is zero.

The National University of Peking 